\title{
LA ENSEÑANZA DE ESPECIES NO CARISMÁTICAS INVERTEBRADAS EN EL CONTEXTO DE LA ESCUELA NUEVA:UN ANÁLISIS DESDE LOS LIBROS DETEXTO
}

\section{Teaching of Non-Charismatic Invertebrate Species in the Context of the New School: An Analysis Based on Textbooks}

\section{O ensino de espécies não carismáticas invertebradas no contexto da escola nova: uma análise desde os livros de texto}

\author{
Nidia Yaneth Torres-Merchán ${ }^{1}$ \\ Marisol Rojas-Niño ${ }^{2}$
}

Fecha de recepción: 12 de octubre de 2016 Fecha de aceptación: 8 de junio de 2017

\section{Resumen}

Este artículo describe los principales resultados de una investigación que tiene como objetivo el análisis de la enseñanza de especies no carismáticas, específicamente los invertebrados, en libros de texto utilizados por docentes de algunas sedes rurales de la institución educativa del municipio de Floresta (Boyacá). El escrito recoge las concepciones de doce profesores sobre la enseñanza del tema, también se analizó un total de 36 libros de texto, incluyendo las cartillas de autoaprendizaje de Escuela Nueva, dadas por el Ministerio de Educación Nacional, y algunos textos complementarios usados por los docentes, desde los grados segundo a quinto de educación básica. Se analizaron aspectos como explicación del tema, actividades desarrolladas, porcentaje de páginas dedicadas al estudio, presencia de conceptos y jerarquías textuales. Como resultado se encontró que la enseñanza de especies no carismáticas es abordado desde temas como reproducción, ecosistemas, seres vivos, conservación de la biodiversidad. También, se observó que las imágenes de invertebrados son utilizadas de manera antropomorfizada y alrededor del $23 \%$ de las páginas solo mencionan ejemplos de estas; así mismo, se observa que en los textos se hace énfasis en los insectos como especies que trasmiten enfermedades.

Palabras clave: especies no carismáticas; escuela nueva; educación científica

1 Doctora en Didáctica de las ciencias experimentales, profesora Facultad de Educación, Universidad Pedagógica y Tecnológica de Colombia, Investigadora grupo WAIRA, nidia.torres@uptc.edu.co

2 Magister en Educación, Licenciada en Ciencias Naturales y Educación Ambiental. Semillero de investigación grupo WAIRA, Universidad Pedagógica y Tecnológica de Colombia, marisol.rojasnino@uptc.edu.co 


\section{Abstract}

This article describes the main results of a research intended to analyze the teaching of non-charismatic species, more specifically of invertebrates, in textbooks used by school teachers from the municipality of Floresta (Boyacá). The paper gathers the views of 12 teachers about teaching this topic, and a total of 36 textbooks are analyzed, including the Escuela Nueva self-learning booklets provided by the Ministry of Education of Colombia and a few supplementary texts used by teachers from second to fifth grade of basic education. Aspects such as the introduction of the subject, activities developed, percentage of pages dedicated to the study, presence of concepts, and textual hierarchies were analyzed. Results show that the teaching of non-charismatic species is approached from other topics such as reproduction, ecosystems, living beings, conservation of biodiversity. The study also shows that the images of invertebrates are used in an anthropomorphized way, and approximately $23 \%$ of the pages only mention examples; it is also seen that the textbooks emphasize on insects as disease transmitting species.

Keywords: non-charismatic species; new school; scientific education

\section{Resumo}

Este artigo descreve os principais resultados de uma pesquisa que visa analisar o ensino de espécies não carismáticas, especificamente dos invertebrados, em livros de texto utilizados por professores de algumas escolas de campo do município de Floresta, Boyacá. $O$ texto apresenta as concepções de 12 professores sobre o ensino do tema, também analisaram-se 36 livros de texto em total, incluindo as cadernetas de autoaprendizagem de Escola Nova, contribuição do Ministério da Educação Nacional, e alguns textos complementares utilizados pelos professores desde o segundo até o quinto ano de educação básica. Analisaram-se aspectos como explicação do tema, atividades desenvolvidas, porcentagem de páginas dedicada aos estudos, presença de conceitos e hierarquias textuais. Como resultado, evidenciou-se que o ensino de espécies não carismáticas é abordado desde temáticas como reprodução, ecossistemas, seres vivos, preservação da biodiversidade. Também, observou-se que as imagens de invertebrados são utilizadas de forma antropomorfizada e ao redor de $23 \%$ das páginas só utilizam esses exemplos. Assim mesmo, observou-se que nos textos se faz ênfase nos insetos como espécies que transmitem doenças.

Palavras-chave: espécies não carismáticas; escola nova; educação científica 


\section{Introducción}

El estudio de la biodiversidad para la conservación ha sido uno de los temas con mayor relevancia en el aprendizaje de las ciencias (Campos et al., 2012). Distintas investigaciones indican que se hace más énfasis en la conservación de los vertebrados y un desconocimiento sobre la función biológica que pueden tener especies invertebradas (Matthews, Flage y Matthews, 1997; Mckinney, 2002; Samways, 2005; Losey y Vaughan, 2006; New, 2011; Prokop et al., 2016).

Esta distinción sobre conservación de especies que tienden a ser atractivas para el público es base del concepto de especies carismáticas (Heywood, 1995, citado por Arango, Rozzi, Massardo, Anderson e lbarra, 2007), mientras que las no carismáticas son las especies que por su aspecto no son llamativas para los programas de conservación y protección animal, pese a su importancia biológica (Jones, Bateman, Kontoleon, Ferrini, Burgess y Turner, 2012).

La importancia biológica de las especies no carismáticas es relativamente amplia, en cuanto llevan a cabo la mayor parte de los servicios en los ecosistemas (Mckinney, 2002; Snaddon, Turner y Foster, 2008). Estas especies son una potente herramienta para la promoción de especies de bajo perfil con alto valor de conservación biológica dentro de las aulas de clase (Root-Bernstein, Douglas, Smith, Veríssimo, 2013).

Entre los estudios sobre percepciones de niños acerca de especies no carismáticas se destacan el de Ballouard, Provost, Barré y Bonnet (2012), quienes evaluaron el impacto que causa la manipulación de algunas serpientes. Los autores proponen que las experiencias de campo permiten el contacto físico con los animales y este es un elemento crucial para mejorar la actitud de los estudiantes hacia un organismo impopular.

Así mismo, Prokop et al. (2016) evalúan la tolerancia a especies como las ranas, y revelan que la emoción de disgusto se correlaciona negativamente con la voluntad de proteger a varios animales. Por otra parte, Dikmenli, Çardaky Öztaş (2009) demuestran que los alumnos generalmente desarrollan concepciones alternativas como resultado de su propia interpretación o de algunas explicaciones contradictorias en la escuela o fuera de entornos escolares en primeros grados.

Torres y Medina (2014) describen las consideraciones que tienen los estudiantes de educación básica primaria de instituciones educativas acerca de especies carismáticas y no carismáticas. Se encontró que los participantes prefieren animales domésticos y exóticos; de igual forma fundamentan sus preferencias sobre alguna característica estética acerca de los animales, así como apreciaciones en cierta utilidad. Este mismo estudio proyecta la necesidad de efectuar un análisis sobre cómo es abordado el concepto especies no carismáticas en el contexto de la educación primaria, pues el análisis de libros de texto se constituye en una herramienta que ayuda al docente a ser crítico para poder discriminar aquellos materiales que no pueden ser útiles o complementar la enseñanza con otro tipo de herramientas educativas (Bermúdez, De Longhi, Díaz y Gavidia, 2014; Gil y de la Gándara, 1995; Guerra y López 2011; Henno y Reiska, 2010; Jiménez, 2013; López y Guerra, 2013; Mazas y Fernández, 2016; Robinson, Fischer, Wiley y Hilton, 2014). Por ende, el análisis de este material didáctico permite dilucidar la necesidad de apropiar herramientas metodológicas que permitan orientar sobre cómo debe enseñarse las especies no carismáticas invertebradas, esto para evitar la generación de actitudes negativas frente a ellas.

El conocimiento referente a la enseñanza de las ciencias que es impartido por las instituciones educativas, se transmite por medio de los libros de texto, que constituyen una ayuda inestimable para el profesor en el trabajo diario del aula, pues ofrecen una concepción legitimada del saber a enseñar (Bermúdez et al., 2014). Una muestra de la importancia que se le da al libro de texto en la enseñanza de las ciencias es que este se ha convertido en objeto de estudio para los investigadores en la didáctica de las ciencias (Solaz, 2010), por cuanto contiene elementos pedagógicos y didácticos para el abordaje y la enseñanza-aprendizaje de los elementos a enseñar.

De este modo, al analizar los contenidos de los libros de texto en el contexto de los conocimientos biológicos, se pueden llegar a considerar aspectos importantes para que la enseñanza de las ciencias en el aula fomente ambientes de aprendizaje de los estudiantes. En este caso, los contenidos acerca de especies no carismáticas (invertebrados), en cuanto estas constituyen una base del conocimiento de las ciencias en primaria, que influirá de manera determinante en aspectos con respecto al papel biológico en el ambiente.

En este sentido, como lo indica Gracia (2003), los libros de texto pueden llegar a generar algunas ideas erróneas o pueden no involucrar temas específicos básicos en la formación de estudiantes en ciencias. Por ello, este escrito tiene como objetivo analizar cómo el tema de especies no carismáticas, en especial los invertebrados, está siendo abordado en los libros de texto, aspecto que permite hacer reflexiones interesantes sobre la necesidad de 
diseñar material didáctico para el abordaje de este tema desde los escenarios de educación primaria.

\section{Materiales y métodos}

Para el desarrollo de esta investigación se consideraron dos momentos: el primero, orientado a conocer el tipo de libros de texto que utilizan los profesores de básica primaria para la enseñanza de las ciencias, mediante una entrevista semiestructurada dirigida a 12 de los docentes de instituciones educativas rurales del municipio de Floresta (Boyacá, Colombia), quienes fueron informados del propósito de la investigación. Este instrumento fue diseñado con base en el estudio doctoral de Calderero (2003), las preguntas fueron adaptadas de acuerdo con el objeto de este estudio (tabla 1).

Tabla 1. Cuestionamientos presentados a los docentes participantes

\begin{tabular}{|c|c|c|}
\hline Cuestionamientos & Propósito & Aspectos de análisis \\
\hline $\begin{array}{l}\text { 1. ¿Cómo considera el uso del libro de texto en la } \\
\text { enseñanza de las ciencias? }\end{array}$ & $\begin{array}{l}\text { Conocer la importancia de los libros de texto en } \\
\text { la enseñanza de las ciencias. }\end{array}$ & \multirow{2}{*}{ Tipos de libros utilizados } \\
\hline $\begin{array}{l}\text { 2. ¿Qué libros de texto utiliza en la enseñanza de las } \\
\text { ciencias? }\end{array}$ & $\begin{array}{l}\text { Identificar los libros de texto utilizados en la } \\
\text { enseñanza de las ciencias. }\end{array}$ & \\
\hline $\begin{array}{l}\text { 3. ¿Sabe qué son las especies no carismáticas? ¿Podría } \\
\text { dar una definición? }\end{array}$ & $\begin{array}{l}\text { Identificar el concepto que tienen acerca de las } \\
\text { especies no carismáticas. }\end{array}$ & \multirow[b]{2}{*}{$\begin{array}{l}\text { Conocimientos frente al tema de } \\
\text { especies no carismáticas }\end{array}$} \\
\hline $\begin{array}{l}\text { 4. ¿Aborda en el desarrollo de sus clases los contenidos } \\
\text { referentes a especies no carismáticas? ¿Cómo? }\end{array}$ & $\begin{array}{l}\text { Reconocer si se enseñan contenidos de } \\
\text { especies no carismáticas dentro del aula y } \\
\text { además identificar posibles metodologías de } \\
\text { enseñanza. }\end{array}$ & \\
\hline $\begin{array}{l}\text { 5. ¿Tienen en cuenta los libros de texto que usted utiliza } \\
\text { el tema de especies no carismáticas? }\end{array}$ & $\begin{array}{l}\text { Reconocer si los libros de texto tienen en } \\
\text { cuenta contenidos referentes a especies no } \\
\text { carismáticas. }\end{array}$ & \multirow{3}{*}{ Aspectos metodológicos } \\
\hline $\begin{array}{l}\text { 6. ¿Cree que los contenidos de especies no carismáticos } \\
\text { en los libros de texto son aptos para los estudiantes de } \\
\text { ese grado? ¿Por qué? }\end{array}$ & $\begin{array}{l}\text { Averiguar si los contenidos de los libros de } \\
\text { texto respecto a especies no carismáticas son } \\
\text { apropiados para los estudiantes. }\end{array}$ & \\
\hline $\begin{array}{l}\text { 7. ¿Los contenidos de los libros muestran metodologías } \\
\text { aptas para el abordaje de los conceptos de especies no } \\
\text { carismáticas en la educación básica primaria? }\end{array}$ & $\begin{array}{l}\text { Conocer metodologías citadas por los } \\
\text { libros para el abordaje de los contenidos } \\
\text { relacionados con especies no carismáticas. }\end{array}$ & \\
\hline
\end{tabular}

En un segundo momento, se hizo un análisis de los libros de texto utilizados por los docentes; se tomó una muestra de 36 libros, que son guías de autoaprendizaje de la fundación Volvamos a la Gente, enviados por el Ministerio de Educación Nacional (MEN), y algunos textos complementarios señalados por los docentes; se revisó cada página con el objetivo de identificar cómo eran presentados los conceptos de especies no carismáticas invertebradas. A continuación, se relaciona el nombre de los libros encontrados (tabla 2).

Tabla 2. Libros utilizados para el análisis de especies no carismáticas

\begin{tabular}{|c|c|c|c|c|}
\hline Nombre & Autor & Editorial & Año & Cantidad \\
\hline $\begin{array}{l}\text { Ciencias naturales y medio ambiente ( } 2 \text {, } \\
3,4,5 \text { grado), primera, segunda y tercera } \\
\text { cartilla. }\end{array}$ & $\begin{array}{l}\text { Fundación Multitaller de la } \\
\text { Universidad del Valle }\end{array}$ & $\begin{array}{l}\text { Ministerio de } \\
\text { Educación Nacional }\end{array}$ & 1996 & 12 \\
\hline $\begin{array}{l}\text { Ciencias naturales y educación ambiental } \\
(2,3,4,5 \text { grado), primero y segundo } \\
\text { módulo. }\end{array}$ & $\begin{array}{l}\text { Fundación Escuela Nueva Volvamos } \\
\text { a la Gente }\end{array}$ & $\begin{array}{l}\text { Ministerio de } \\
\text { Educación Nacional }\end{array}$ & 2008 & 8 \\
\hline Camino a la ciencia (1 y 2 ) & $\begin{array}{l}\text { Héctor Guillermo Sierra, Carlos Arturo } \\
\text { Gelves, Fidel Antonio Cárdenas }\end{array}$ & Intermedio Editores & 1988 & 2 \\
\hline $\begin{array}{l}\text { Ingéniate } 3 \\
\text { Ciencias naturales y de la salud }\end{array}$ & $\begin{array}{l}\text { William Gómez, Humberto Caicedo } \\
\text { López, Miguel Ángel Gómez, Edith } \\
\text { Figueredo de Urrego }\end{array}$ & Voluntad & 1989 & 1 \\
\hline
\end{tabular}




\begin{tabular}{|l|l|l|c|c|}
\hline \multicolumn{1}{|c|}{ Nombre } & \multicolumn{1}{c|}{ Autor } & \multicolumn{1}{c|}{ Editorial } & Año & Cantidad \\
\hline Reino mágico (2, 3, 4, 5) & $\begin{array}{l}\text { Guillermo Rojas Rodríguez, Rubén } \\
\text { Darío Bolívar Sepúlveda }\end{array}$ & Educar editores & 1984 & 4 \\
\hline Enciclopedia Juvenil Océano & Grupo Editorial Océano & Océano & 1996 & 4 \\
\hline Enciclopedia Océano de la Ecología & Grupo Editorial Océano & Océano & 1998 & 2 \\
\hline Ciencias naturales (3,4 y 5) & $\begin{array}{l}\text { Yolanda Alegría Melo, Judith Helena } \\
\text { Arteta }\end{array}$ & Santillana & 1989 & 3 \\
\hline
\end{tabular}

En este artículo solo se presentará el análisis realizado a las cartillas de autoaprendizaje de Escuela Nueva, el cual se desarrolló de acuerdo a tres categorías basadas en el estudio hecho por Franco, Castellanos y Medellín (2010) y Occelli, Valeiras y Bernardello (2015).
Categoría 1. Análisis de contenido de las cartillas de Escuela Nueva: Se realizó de acuerdo a las siguientes subcategorías (tabla 3).

Tabla 3. Subcategorías utilizadas para el análisis de las guías de aprendizaje de Escuela Nueva

\begin{tabular}{|c|c|c|c|}
\hline $\begin{array}{l}\text { Sub- } \\
\text { categoría }\end{array}$ & & Criterio & Propósito \\
\hline \multirow{3}{*}{ 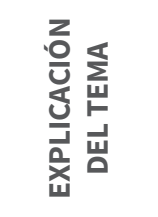 } & \multicolumn{2}{|c|}{ Coherencia: Contenido vs. Objetivos generales y específicos. } & $\begin{array}{l}\text { Analizar coherencia entre los contenidos con los } \\
\text { objetivos planeados. }\end{array}$ \\
\hline & \multicolumn{2}{|c|}{ Coherencia lógica del texto. } & Analizar coherencia entre los contenidos. \\
\hline & \multicolumn{2}{|c|}{ Análisis del lenguaje utilizado. } & $\begin{array}{l}\text { Identificar si se utiliza un lenguaje común o un } \\
\text { lenguaje científico. }\end{array}$ \\
\hline \multirow{4}{*}{ 出 } & \multicolumn{2}{|c|}{ Actividades adecuadas de refuerzo. } & Reconocer tipos de actividades de refuerzo. \\
\hline & \multicolumn{2}{|c|}{ Coherencia del contenido con las actividades prácticas. } & $\begin{array}{l}\text { Analizar coherencia entre los contenidos y las } \\
\text { actividades prácticas. }\end{array}$ \\
\hline & \multirow{2}{*}{ 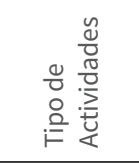 } & $\begin{array}{l}\text { Actividades de papel y lápiz: preguntas, problemas, } \\
\text { ejercicios, gráficos, etc. }\end{array}$ & \multirow{2}{*}{ Identificar tipo de actividades presentes. } \\
\hline & & $\begin{array}{l}\text { Actividades experimentales: experimentos e } \\
\text { investigaciones. }\end{array}$ & \\
\hline \multirow{7}{*}{ 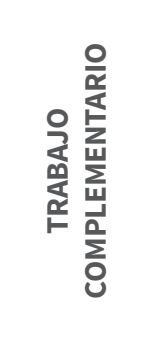 } & \multicolumn{2}{|c|}{ Actividades que involucran el desarrollo de proyectos. } & \multirow{3}{*}{$\begin{array}{l}\text { Reconocer tipo de actividades propuestas como } \\
\text { complemento. }\end{array}$} \\
\hline & \multicolumn{2}{|c|}{ Actividades que involucran el conocimiento de personas externas. } & \\
\hline & \multicolumn{2}{|c|}{ Actividades de socialización. } & \\
\hline & \multirow{4}{*}{ 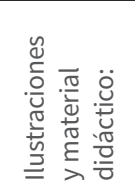 } & Dibujos & \multirow{4}{*}{$\begin{array}{l}\text { Identificar los tipos de dibujos, gráficos, imágenes y } \\
\text { tablas y su importancia en el libro de texto. }\end{array}$} \\
\hline & & Gráficos & \\
\hline & & Fotografías & \\
\hline & & Tablas & \\
\hline
\end{tabular}

Fuente: Adaptado de Franco, Castellanos y Medellín (2010) y Occelli, Valeiras y Bernardello (2015).

Categoría 2. Presencia de los conceptos en las guías de aprendizaje de Escuela Nueva: Se identificó la existencia de contenidos en estas cartillas, respecto a especies no carismáticas invertebradas.

Categoría 3. Jerarquías textuales en las guías de aprendizaje de Escuela Nueva: Se analizó la frecuencia de conceptos relacionados a las especies no carismáticas en cada uno de los textos analizados.
De igual forma, se analizó el porcentaje de páginas dedicadas al estudio de especies no carismáticas. Se realizó teniendo en cuenta la cantidad de presencia de los conceptos en las guías de aprendizaje de Escuela Nueva, estas se subdividen en secciones y guías por cada unidad temática y se identificó en qué sección se encontraba la mayor cantidad de este tipo de especies. 


\section{Resultados y discusión}

Teniendo en cuenta lo propuesto en la metodología, a continuación se presenta el primer momento orientado a identificar el tipo de libros de texto que utilizan los docentes de la educación primaria con respecto a la enseñanza de especies no carismáticas, así como el conocimiento que tienen los profesores sobre estas especies.

\section{Uso de libros de texto en la enseñanza de las ciencias}

Los docentes afirman que el uso de los libros de texto en la enseñanza de las ciencias es importante, debido a que los contenidos contribuyen a la contextualización de situaciones, y permiten de esta manera la apropiación de contenidos y la posible aplicación de los mismos en la vida cotidiana. Por otro lado, los docentes señalan que el libro de texto es un recurso valioso en la enseñanza, debido a que contribuyen al fomento del aprendizaje en el aula.

Se destacan algunas afirmaciones, por ejemplo, el docente D6 señala:

Pues el área de las ciencias es el área más enriquecedora en la parte práctica, el libro es un material de apoyo, pero no es esencial. (Docente D6).

Pues me parece muy interesante porque es una ayuda didáctica para nosotros, a parte del internet, nosotros también tenemos que estar actualizándonos, me parecen textos muy bonitos, para que los niños realicen, trabajen, tienen sopa de letras, tienen crucigramas. (Docente D5).

Estas afirmaciones evidencian que el libro de texto es un recurso para complementar las clases.

Por consiguiente, los doce docentes entrevistados coinciden en decir que los textos del MEN son muy completos y presentan metodologías aptas para el aprendizaje de las ciencias y áreas afines, por lo cual D12 afirma:

\begin{abstract}
Los libros que nosotros manejamos son los libros de Escuela Nueva, elaborados por la fundación volvamos a la gente, son libros muy completos que abordan todos los temas, que se dan en primaria, tienen buena metodología, tienen experimentos con los cuales los chicos aprenden.
\end{abstract}

La entrevista también permitió conocer que los docentes utilizan los textos enviados por el MEN, como libros reglamentarios, titulados Ciencias naturales y educación ambiental y Ciencias naturales y medio ambiente. Los primeros están compuestos por dos módulos y los segundos, por 3 cartillas para cada grado específico. Además, se evidencia que algunos de los docentes se apoyan en otros textos, por ejemplo, enciclopedias y libros de editoriales como Voluntad, Santillana, entre otros.

\section{Conceptos de especies no carismáticas}

En las preguntas específicas sobre las concepciones que tienen los docentes acerca de especies no carismáticas, se encuentra que la mayoría de los docentes no tienen claridad sobre el concepto de especies no carismáticas, pero un docente hace inferencia del concepto y comenta lo siguiente:

La verdad el término, no estoy muy acostumbrada a manejarlo dentro de mi diario de desempeño, pero supongo que cuando hablamos de "carisma" es cuando hay como una afinidad con este tipo de cosas, y las especies no carismáticas son las que poco se requiere estudiar, entonces, por ejemplo los ratones, las ratas, las cucarachas. (Docente D8).

Por otra parte, D7 afirma:

Con el título de especies no carismáticas no, pero lo que pasa es que esas especies van incluidas en cada uno de los rangos de las especies que cada uno estudia, entonces por ejemplo las algas están dentro de los protistas, bueno, en las diferentes clases de animales que se estudian ahí van incluidos los no carismáticos. (Docente D7).

Por ello, las temáticas incluidas en lo referente a los conceptos de especies no carismáticas no se enseña como un tema específico, sino que se ve como algo implícito en otras temáticas.

Sin embargo, al hacer la pregunta de manera distinta, por ejemplo, "¿incluye usted dentro de sus temas de clase la importancia sobre la conservación de invertebrados?", los profesores reconocen que este no es trabajado de forma significativa en el aula. Por tanto, los significados de la enseñanza de las especies no carismáticas pueden depender en gran medida de la información que brindan los libros de texto, y es por ello que resulta importante indagar su contenido (Occelli et al., 2015).

Frente a la pregunta: " ¿Incluye usted dentro de sus temas de clase la conservación de insectos?", y “icómo lo aborda?", los docentes coinciden en decir que esos temas vienen en las guías de texto y de acuerdo con ello son vistos en el aula. Esto indica que los libros de texto, en este caso guías de aprendizaje enviadas por el men, tienen 
en cuenta los contenidos de especies no carismáticas de manera implícita, es decir, que se incluyen dentro de otras temáticas. Esto apoyado en la afirmación de D7:

Los libros las incluyen, generalmente cuando se ven las cadenas alimenticias o cuando se ve la clasificación de los seres de la naturaleza. (Docente D7).

Los docentes consideran que las guías de aprendizaje enviadas por el MEN son adecuadas para la enseñanza de las ciencias y que en especial se enseña el tema de insectos; sin embargo, difieren en que hace falta profundizar su enseñanza:

En la medida en que las actividades son específicas, pero no hay una profundización en saber qué tipo de especies son, no se ha hecho como una clasificación donde al estudiante se le pueda indicar o se le pregunte, ¿niño, sabe cuáles son las especies no carismáticas?, él no va a saber cuáles son, y es precisamente por eso, no porque no haya sido estudiado, sino porque no sabe su clasificación. (Docente D7).

Los docentes indican que como son guías propuestas por el men los contenidos para cada uno de los grados tienen que ir acorde con los estándares mínimos y las competencias en ciencias naturales. Las guías de aprendizaje, además de traer los contenidos específicos para los estudiantes, traen metodologías aptas para el estudio:

La metodología planteada en las guías de autoaprendizaje vienen específicas, hay mucha actividad práctica y también se nos da la oportunidad de hacer la adaptación de guías, pero sería bueno que nosotros que trabajamos todas las áreas, pudiéramos tener a una persona especializada en el tema que lo asesore, esto ayuda a profundizar los contenidos. (Docente D3).

\section{Análisis de los libros de texto}

De acuerdo con los resultados de la entrevista, se analizaron las guías de aprendizaje de Escuela Nueva de la fundación Volvamos a la Gente (1996, 2008), en ellas se revisaron de manera detallada en qué parte de las guías se desarrollaban los contenidos referentes a especies no carismáticas de acuerdo con las siguientes categorías:

\section{Categoría 1. Análisis de contenido de las cartillas de Escuela Nueva}

A continuación, se presenta un análisis de los contenidos didácticos abarcados en las guías de aprendizaje de los años 1996 y 2008, en este se encuentran tres secciones: la primera corresponde a la explicación del tema y los respectivos criterios para su análisis; la segunda, a las actividades que se desarrollan teniendo en cuenta el tipo de actividades, y, por último, hay una sección de trabajo complementario con sus respectivos criterios de análisis (tabla 3).

\section{Explicación del tema}

En esta sección, se reconoce la forma como se abarca la temática principal de las especies no carismáticas en los libros de texto abordados. Se analizó si el contenido presenta aspectos conceptuales sobre el tema. De acuerdo con esto, se analizan los siguientes criterios:

\section{Coherencia: contenido vs. objetivos generales y específicos}

En relación con la estructura general de los textos, se encontró que todas las guías de aprendizaje presentan los objetivos mencionados como logros, en cada una de las temáticas y al inicio de cada unidad. Las guías de aprendizaje Escuela Nueva 2008 se subdividen en tres secciones:

- La primera sección se caracteriza por presentar factores comunes enfocados en el aprendizaje de las ciencias naturales.

- En la segundad sección, se proponen actividades de práctica enfocadas en el desarrollo de actividades adicionales por parte de los estudiantes, con el apoyo de los docentes y, en otras ocasiones, a partir del trabajo autónomo, mientras que en las guías de texto 1996 prima la lectura como forma de enseñanza.

- En la tercera sección se plantean actividades para solucionar con la familia.

Al revisar los elementos conceptuales que se tienen en cuenta para el desarrollo de la temática propios de las ciencias naturales, hay un orden en los contenidos basado en los estándares básicos. Se observa además que el tratamiento de contenidos referidos a especies no carismáticas invertebradas no se abarca solo, sino que está implícito en otras temáticas, por ejemplo, en reproducción y relaciones de los seres vivos, entre otros. Al respecto se esperaría que las guías propusieran actividades que impliquen la experiencia directa con estas especies, pues, como lo sugieren estudios de Ballouard et al. (2012) y Prokop et al., (2016), la experiencia biológica de un niño influye en conceptos sobre los organismos vivos. También, como estas guías están dirigidas a población rural, es importante tener en cuenta este aspecto como situación favorable para examinar el efecto de las experiencias personales sobre sus conocimientos. 


\begin{tabular}{|c|c|c|c|c|c|c|c|c|c|c|c|c|c|c|}
\hline$\underset{\infty}{\mathcal{\infty}}$ & 2 & $\bar{s}$ & uṇuor & is & is & is & is & 운 & is & is & is & $i \bar{s}$ & is & is \\
\hline $\overrightarrow{0}$ & $\stackrel{0}{z}$ & $\bar{s}$ & uṇuor & is & is & is & is & 운 & is & $i \bar{s}$ & $\overline{i n}$ & is & is & is \\
\hline 옹 & 2 & $i$ & uṇuor & is & is & is & is & 2 & is & $i \bar{s}$ & is & is & is & is \\
\hline 오 & $\stackrel{0}{z}$ & is & uñuo & is & is & in & is & 운 & is & is & is & is & is & is \\
\hline$\stackrel{\infty}{\infty}$ & $\stackrel{0}{z}$ & $i$ & unֻuo & is & is & is & is & 2 & is & $\overline{i n}$ & is & is & is & is \\
\hline$\tilde{\infty}$ & 2o & is & uṇuos & is & is & is & is & 운 & is & is & is & $i$ & $i \bar{s}$ & is \\
\hline $\mathscr{\bullet}$ & zo & is & uṇmos & is & is & is & is & 우 & is & is & is & is & is & is \\
\hline 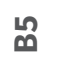 & 을 & is & uñuo & $i \bar{s}$ & is & is & is & 은 & is & is & is & is & $\overline{i s}$ & is \\
\hline Ф & $\stackrel{\circ}{z}$ & is & uñuo & is & is & is & is & $\frac{0}{2}$ & is & is & is & $\bar{\omega}$ & is & i \\
\hline $\mathscr{\mathscr { D }}$ & 을 & is & uṇmos & i & is & is & is & 우 & is & is & $\bar{n}$ & in & $i \bar{s}$ & is \\
\hline$\widetilde{\infty}$ & zo & is & uñuo & is & is & is & is & \& & is & is & is & $i \bar{s}$ & is & is \\
\hline$\vec{\infty}$ & $\stackrel{\circ}{2}$ & is & uñuo & is & is & is & is & $\stackrel{0}{z}$ & is & is & $i$ & is & is & is \\
\hline$\stackrel{\infty}{<}$ & $\bar{n}$ & is & uñuo & is & is & is & is & 운 & is & is & is & $i \bar{s}$ & is & is \\
\hline 疋 & $\bar{n}$ & is & uṇuoJ & is & is & is & 2 & 운 & is & $i \bar{s}$ & is & is & is & is \\
\hline $\mathscr{\alpha}$ & is & is & uñuo & is & is & is & 우 & 운 & is & is & $\bar{n}$ & is & $i \bar{s}$ & is \\
\hline 足 & $\bar{n}$ & is & uñuo & is & is & is & 운 & 은 & is & is & is & is & is & is \\
\hline \& & i & is & uñuo & i & is & is & 운 & 은 & is & is & is & in & is & is \\
\hline$\stackrel{m}{<}$ & in & in & uñuo & is & in & is & 운 & $\stackrel{\circ}{z}$ & is & is & in & is & $\overline{i s}$ & is \\
\hline ָ̃ & is & is & uṇuos & $i \bar{s}$ & is & is & 운 & 우 & is & is & $\bar{n}$ & $i \bar{s}$ & $i \bar{s}$ & is \\
\hline 安 & is & $\bar{n}$ & uñuos & i & is & is & 우 & 을 & is & is & $\bar{n}$ & $\bar{s}$ & $\bar{n}$ & is \\
\hline $\begin{array}{l}\text { 足 } \\
\stackrel{\underline{m}}{=}\end{array}$ & 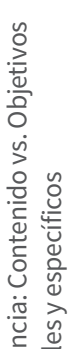 & 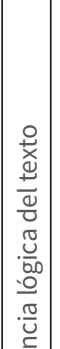 & 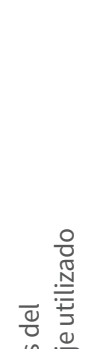 & 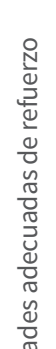 & 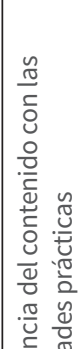 & 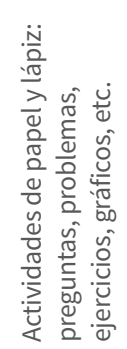 & 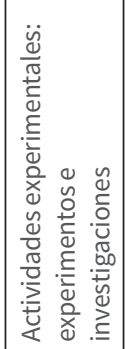 & \multirow{2}{*}{ 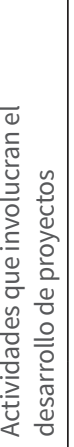 } & \multirow{2}{*}{ 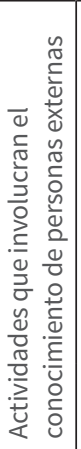 } & \multirow{2}{*}{ 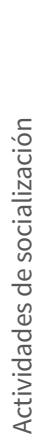 } & $\begin{array}{l}\stackrel{0}{亏} \\
\stackrel{0}{0}\end{array}$ & 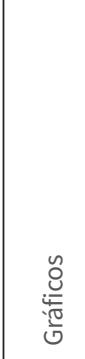 & $\begin{array}{l}\frac{20}{\pi} \\
\frac{5}{00} \\
0 \\
0 \\
0 \\
\end{array}$ & $\frac{\pi}{\frac{\pi}{0}}$ \\
\hline \multirow{2}{*}{ 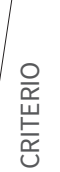 } & 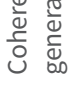 & 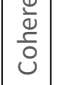 & 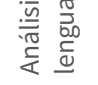 & $\sum_{\bar{Z}}^{\frac{0}{4}}$ & 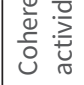 & sәрер!پ! & $\forall \forall$ әр od!! & & & & & رәرе & & \\
\hline & \multicolumn{3}{|c|}{ 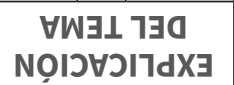 } & \multicolumn{4}{|c|}{ 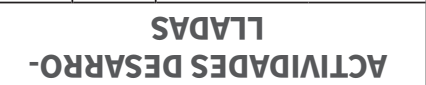 } & \multicolumn{7}{|c|}{ 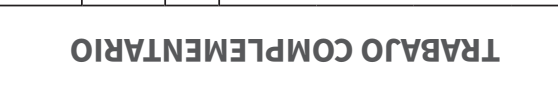 } \\
\hline
\end{tabular}


A1: Ciencias naturales y educación ambiental 2, módulo 1 ; A2: Ciencias naturales y educación ambiental 2, módulo 2; A3: Ciencias naturales y educación ambiental 3 , módulo 1 ; A4: Ciencias naturales y educación ambiental 3 , módulo 2; A5: Ciencias naturales y educación ambiental 4 , módulo 1 ; A6: Ciencias naturales y educación ambiental 4, módulo 2; A7: Ciencias naturales y educación ambiental 5 , módulo 1 ; A8: Ciencias naturales y educación ambiental 5, módulo 2 .

B1: Ciencias naturales y medio ambiente 2, primera cartilla; B2: Ciencias naturales y medio ambiente 2 , segunda cartilla; B3: Ciencias naturales y medio ambiente 2 , tercera cartilla; B4: Ciencias naturales y medio ambiente 3 , primera cartilla; B5: Ciencias naturales y medio ambiente 3 , segunda cartilla; B6: Ciencias naturales y medio ambiente 3 , tercera cartilla; B7: Ciencias naturales y medio ambiente 4, primera cartilla; B8: Ciencias naturales y medio ambiente 4 , segunda cartilla; B9: Ciencias naturales y medio ambiente 4, tercera cartilla; B10: Ciencias naturales y medio ambiente 5 , primera cartilla; B11: Ciencias naturales y medio ambiente 5 , segunda cartilla; B12: Ciencias naturales y medio ambiente 5 , tercera cartilla.

\section{Coherencia lógica del texto}

La articulación de los conceptos es apropiada con respecto a las temáticas a abordar, sin embargo, la extensión que abarca los contenidos de especies no carismáticas es corta, comparada con otros textos escolares. Es por ello que se considera que aún falta vincular temas relacionados con conservación de especies, en cuanto contribuye a formar una mejor visión de los contenidos y puede facilitar la realización de proyectos de conservación de la naturaleza (Schlegel y Rupf, 2010).

\section{Análisis del lenguaje utilizado}

En cuanto al lenguaje utilizado por los textos, es un lenguaje comprensible para los estudiantes. A manera de ejemplo se presenta los siguientes segmentos:

En la naturaleza existe gran cantidad de seres vivos, entre ellos las plantas y los animales. Hay animales grandes y pequeños, terrestres, aeroterrestres, acuáticos y anfibios. (Ciencias naturales y educación ambiental 2, p. 86).

Las relaciones o interacciones entre los organismos vivos cumplen distintas funciones, como son procurar alimentación, brindar y obtener protección, polinización y dominio de espacio. (Ciencias naturales y educación ambiental 2, p. 90).

Se evidencia un lenguaje claro, pero que no profundiza en las funciones biológicas de los organismos. Al respecto, Mateos (1998) señala que es necesario utilizar un lenguaje ajustado a la realidad ecológica del animal y carente de expresiones agresivas, también sugiere que la inclusión de ilustraciones más veraces y menos tendenciosas podría contribuir a tal fin.

\section{Actividades desarrolladas}

Para realizar el análisis de las actividades desarrolladas se tuvieron en cuenta los siguientes criterios:

\section{Actividades adecuadas de refuerzo}

Las actividades que se desarrollan en la guía proponen contestar unos cuestionamientos planteados; sin embargo, hacen falta actividades que fomenten en los estudiantes el interés por la conservación de especies no carismáticas y contribuyan a desarrollar habilidades de pensamiento científico; por lo cual sería interesante que los estudiantes pudieran generar experiencias con la identificación de individuos y se desarrolle algún tipo de trabajo experimental con este tipo de individuos que promueva actitudes de conservación.

\section{Coherencia del contenido con las actividades de práctica}

Los contenidos de cada unidad respecto a las especies no carismáticas están inmersos en contenidos como la reproducción, los ecosistemas, enfermedades; por lo cual las actividades de práctica se convierten en talleres con preguntas, cuadros a completar, descripciones, entre otros. Se evidenció que en las guías de aprendizaje se solicita que los estudiantes den respuestas a cuestionamientos propuestos o ejercicios de descripción, correlación, entre otros. En las guías de aprendizaje 2008, no se fomenta en el estudiante la conservación de especies no carismáticas, mientras que en algunas de las guías de texto 1996, se fomenta en el estudiante una observación del entorno, aunque aún hace falta promover en ellos actitudes de valoración y respeto por los individuos que construyen el ecosistema.

\section{Tipos de actividades}

Todas las guías utilizadas cuentan con actividades para los estudiantes, es por esta razón que se tendrán en cuenta dos tipos de actividades:

\section{Actividades de lápiz y papel (preguntas problema)}

Se evidenció que en las guías de aprendizaje se condiciona al estudiante a dar respuesta a cuestionamientos propuestos o ejercicios de descripción, correlación, entre otros. En las guías de aprendizaje 2008, no se fomenta en el estudiante un espíritu crítico, y así un pensamiento científico, donde surja de ellos la curiosidad por la ciencia 
y los procesos científicos; mientras que, en algunas de las guías de texto 1996, se fomenta en el estudiante una observación del entorno, aunque aún hace falta promover en ellos un espíritu crítico y así un pensamiento científico.

\section{Actividades experimentales (experimentos e investigaciones)}

Cuando se hace referencia a investigaciones no se tuvo en cuenta en el estudio las consultas externas de temas relacionados, sino como experiencias de aula, por ejemplo, salidas de campo, visitas de observación, prácticas de laboratorio, etc., que permitan una contextualización de la temática más enfocada al estudio de las especies no carismáticas.

Dentro de las guías, las únicas actividades que se relacionan fueron algunas salidas al jardín del colegio o alrededores, donde se daban indicaciones para observar el entorno o visitar una quebrada cercana al colegio. Se considera que sería conveniente plantear actividades en las que el estudiante pueda vincularse con problemáticas locales y a la vez proponer posibles soluciones, por ejemplo, análisis de características de individuos invertebrados en la región, investigaciones sobre especies en la región, salidas de campo, visitas a museos, entre otras.

\section{Trabajo complementario}

En este aspecto también se implementó una serie de criterios que constituyen actividades que complementan el trabajo desarrollado en las clases, este tipo de actividades son:

\section{Actividades que involucran el desarrollo de proyectos}

Se evidencia que en las guías de aprendizaje no se promueve el desarrollo de proyectos pues las actividades son dirigidas a responder preguntas propuestas en la guía de trabajo para posteriormente presentarlas al docente. Este tipo de actividades no se incluye en ninguno de los textos, puesto que no se da a considerar el desarrollo de hipótesis o productos educativos que reflejen ingeniar modelos que ejemplifiquen de un modo distinto al planteado en el eje conceptual.

\section{Actividades que involucran \\ el conocimiento de personas externas}

Se evidencia en las secciones $C$ y $D$ de las cartillas el planteamiento de preguntas que involucran asesoría de personas externas a la institución, pero en su mayoría refieren a la familia.

\section{Actividades de socialización}

Dentro de las guías estudiadas se observa que, dependiendo de las actividades propuestas, los estudiantes socializan, realizan trabajos individuales o grupales. Estos procesos contribuyen a la formación del estudiantado, ya que permiten compartir sus experiencias de aula, fomentar sus opiniones o en el simple acto de saber escuchar la opinión de los otros (Franco, Castellanos y Medellín, 2010), puesto que ciertas actividades están sujetas a una interacción dentro del espacio académico.

\section{Ilustraciones y material didáctico}

En la revisión realizada a los libros de texto, se evidenció que existe gran cantidad de ilustraciones en su mayoría de especies carismáticas vertebradas con finalidad decorativa.

En cuanto al estudio de invertebrados, se evidencia que los libros de texto muestran imágenes de animales antropomorfizados, lo cual podría influir en la concepción que adquieren los niños respecto a este tipo de especies animales (Rosenow, 2008). Además, en su mayoría las imágenes son utilizadas como ejemplo visual de representación. A pesar de ello, se observa en ocasiones la presencia de una fotografía, la cual permite contextualizar contenidos biológicos a través de la representación de relaciones entre factores, procesos y estructuras de seres vivos (Robinson et al., 2014).

Es común que las imágenes pertenecientes a invertebrados sean utilizadas para decorar el libro de texto, ya sea un párrafo, una ronda, una fábula, etc. Hacen falta comentarios que indiquen la función que cumple esa imagen en la sección o en el contenido temático. Por otro lado, se hace evidente la falta de gráficas que se vinculen con el tema analizado en los libros objetos de estudio. Sin embargo, las temáticas que presentan estas cartillas respecto a los seres vivos o seres de la naturaleza están acordes con los estándares básicos de ciencias naturales para los grados de educación básica primaria, en cuanto tienen que ser vistos en los respectivos grados. Las actividades que se proponen solucionar en las guías son actividades cortas y fáciles de responder con el fin de fomentar el desarrollo de destrezas en los estudiantes.

Para el caso de las tablas, algunas proveen información general de invertebrados. Por ejemplo, en la tabla de los reinos de la naturaleza, se muestra cada reino, una pequeña descripción y una imagen de un individuo representativo; también sirven como actividad de complemento para desarrollar, ya sea para dibujar o completar, conceptos sobre individuos invertebrados. 
De esta manera, se puede evidenciar que los contenidos referentes a especies no carismáticas se incluyen dentro de los libros de texto (en este caso las guías de aprendizaje), de manera implícita, es decir, que está incluido en algunas temáticas sin que esta lo diga o lo especifique. Por tanto, se comprueba lo que los docentes inicialmente habían afirmado al respecto, en cuanto a que se ven temas referentes a especies no carismáticas, aunque no se nombren en particular, sino que estén involucradas en temáticas como ecosistemas y reinos de la naturaleza.

\section{Categoría 2. Presencia de los conceptos en las guías de aprendizaje de Escuela Nueva}

En función de la presencia de contenidos relacionados con el estudio de las especies no carismáticas invertebradas en los libros, se observó que aquellos que fueron incorporados con mayor frecuencia en las cartillas de 2008 fueron: ecosistemas, clasificación de seres vivos, hábitat, cuidado del medio ambiente, movimiento, enfermedades, adaptación, entre otros (figura 1), mientras en las guías de 1996 los conceptos son reproducción, recursos naturales, relaciones, ecosistemas, entre otros (figura 2).

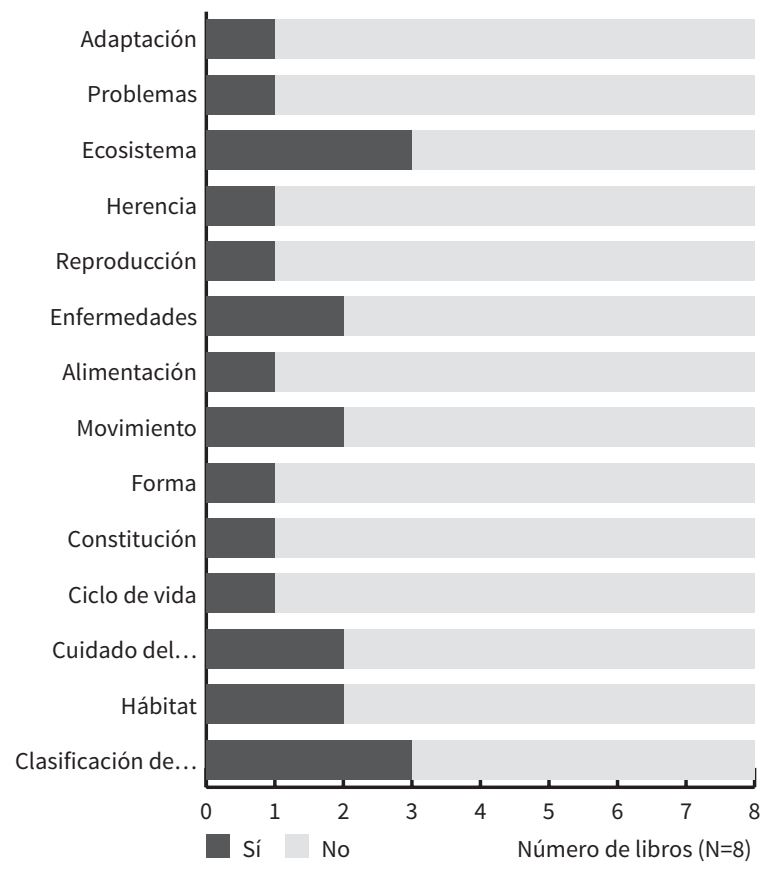

Figura 1. Presencia de los conceptos en las guías de aprendizaje de Escuela Nueva 2008

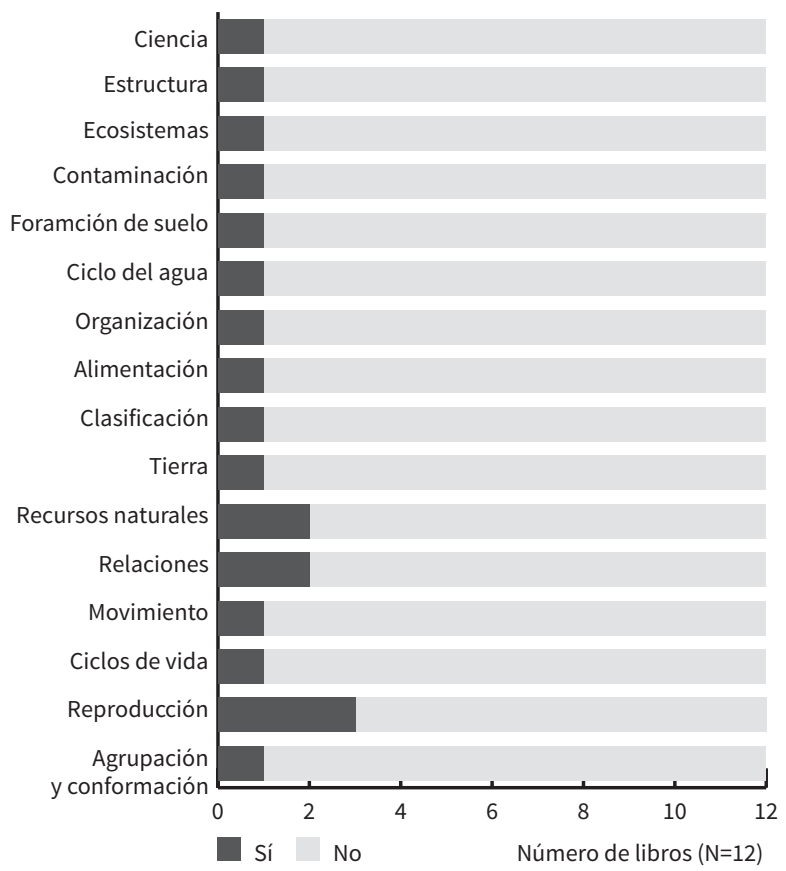

Figura 2. Presencia de los conceptos en las guías de aprendizaje de Escuela Nueva 1996

De este modo, cada guía maneja conceptos diferentes en los que está inmerso el estudio de las especies no carismáticas. Los conceptos sobre estas especies, en su mayoría, no se desarrollan en la unidad principal, sino que son mencionados como complemento del tema principal, de esta manera se muestra su importancia dentro del texto.

Estos resultados a su vez coinciden con los encontrados por Occelli et al. (2015) al analizar el desarrollo de la biotecnología en libros de texto en Argentina. Estos autores concluyen que los conceptos de biotecnología, en general, son abordados en lecturas complementarias ubicadas fuera del texto principal del capítulo, en apartados especiales o en paratextos.

Al respecto, estamos de acuerdo con Bermúdez, De Longhi, Díaz y Gavidia (2014): los libros de texto tratan la temática de manera descontextualizada del ámbito social, aun cuando la dimensión ética y política de la biodiversidad es consustancial a su carácter científico y social. De este modo, se pueden vincular los aspectos que Bermúdez et al. (2014) consideran son relevantes para el estudio de los libros de texto:

a) No contemplan de forma adecuada los conceptos relacionados con el entorno local y más próximo del estudiante.

b) Dan ejemplos de especies carismáticas, como el tigre, el elefante y el oso, pero rara vez de especies invertebradas no carismáticas. 
c) No desarrollan la vertiente social de la problemática medioambiental (enfoque reduccionista).

d) Ilustran ecosistemas idealizados en los que el ser humano se encuentra ausente, salvo como agente de destrucción.

\section{Categoría 3. Jerarquías textuales en las guías de aprendizaje de Escuela Nueva}

En la figura 3 se puede observar una vinculación de las jerarquías textuales de los libros de texto de escuela nueva 2008, es decir, cómo fue abordado cada uno de los conceptos dentro de la guía de aprendizaje. Los conceptos que se desarrollaron con mayor cantidad de elementos contextuales fueron los ecosistemas, debido a que se expuso el tema con ejemplificaciones.

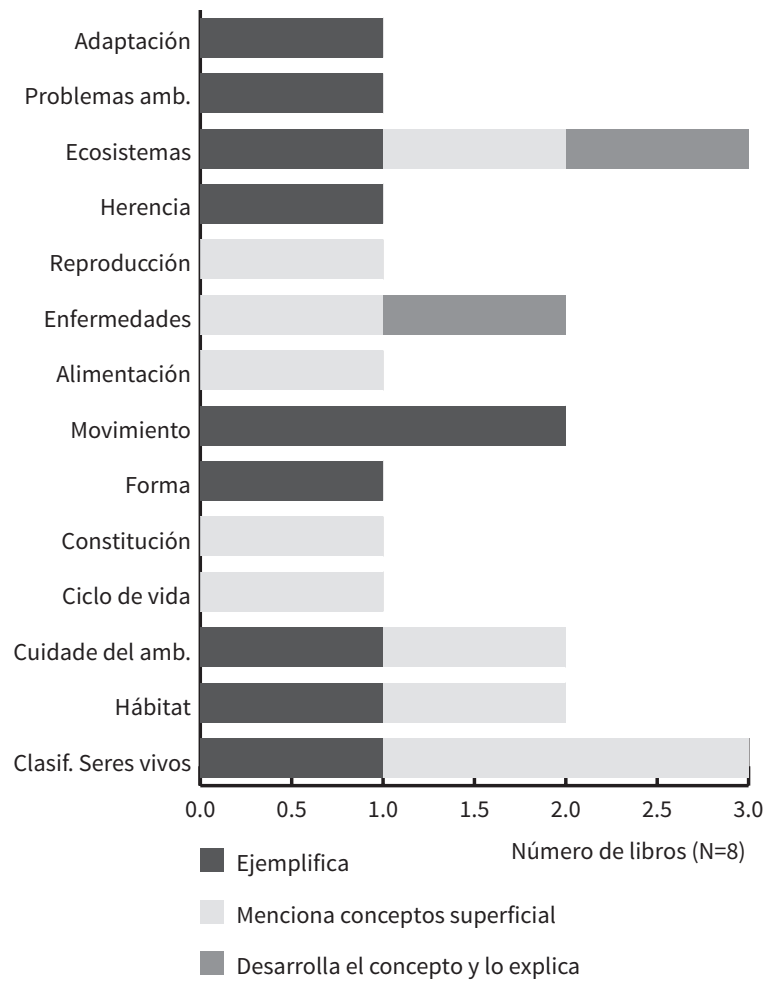

Figura 3. Jerarquía textual de los conceptos en las Guías de aprendizaje de Escuela Nueva 2008

En las cartillas de 1996 (figura 4), los conceptos que se estudiaron con mayor frecuencia fueron recursos naturales y relaciones de los seres vivos. Además, se logra visualizar que se desarrolló el tema con ejemplificaciones e imágenes que hacen que el texto sea un poco más llamativo para los estudiantes de los grados a los que está dirigido.

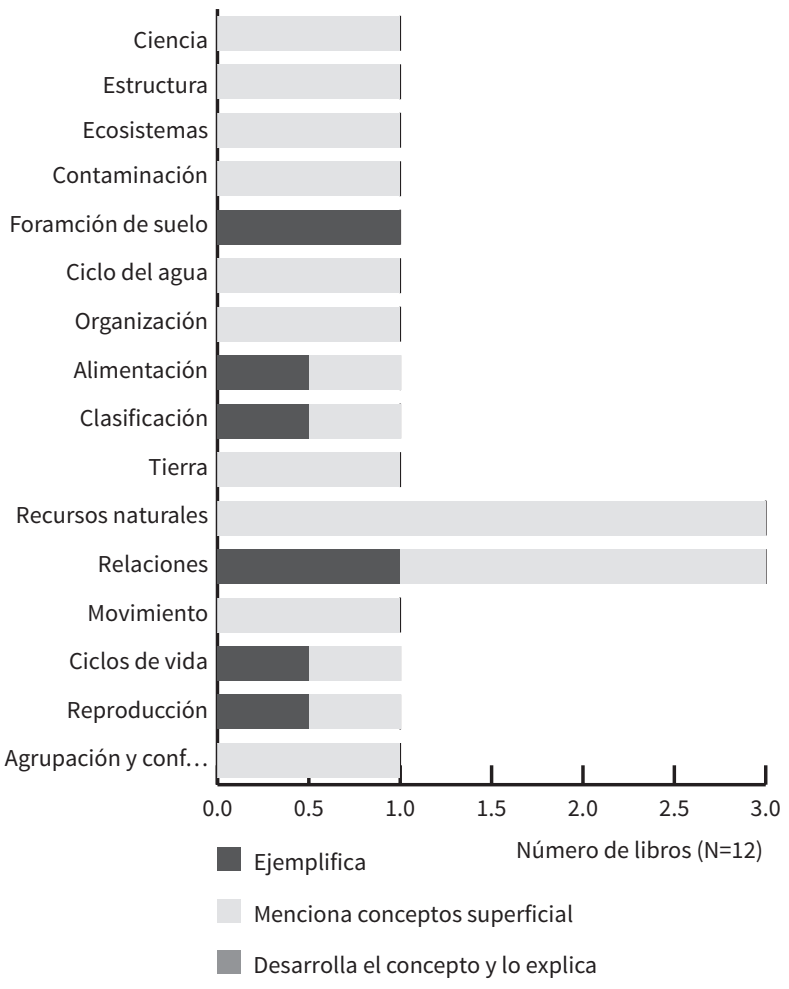

Figura 4. Jerarquía textual de los conceptos en las guías de aprendizaje de Escuela Nueva 1996

\section{Porcentaje de páginas dedicadas al estudio de especies no carismáticas}

Cabe resaltar que dentro de ninguna de las guías de aprendizaje se presenta la palabra "no carismáticos", por ello, para el estudio se tomó en cuenta la presencia de contenidos referidos a invertebrados. Además, se evidencia que la presencia de estos contenidos se dan gracias a un tema central más amplio que lo abarca, por ejemplo, clasificación de seres vivos.

A partir de las figuras 5 y 6 , se puede hacer una comparación entre el porcentaje de páginas dedicadas al estudio de los invertebrados en las guías de aprendizaje de escuela nueva de 2008 y 1996. En las primeras, el mayor porcentaje se encuentra en el módulo 1 de la guía de ciencias naturales 3 , mientras que en las segundas, el mayor porcentaje de páginas se encuentra en la segunda cartilla de la guía de ciencias naturales y medio ambiente 2 . 


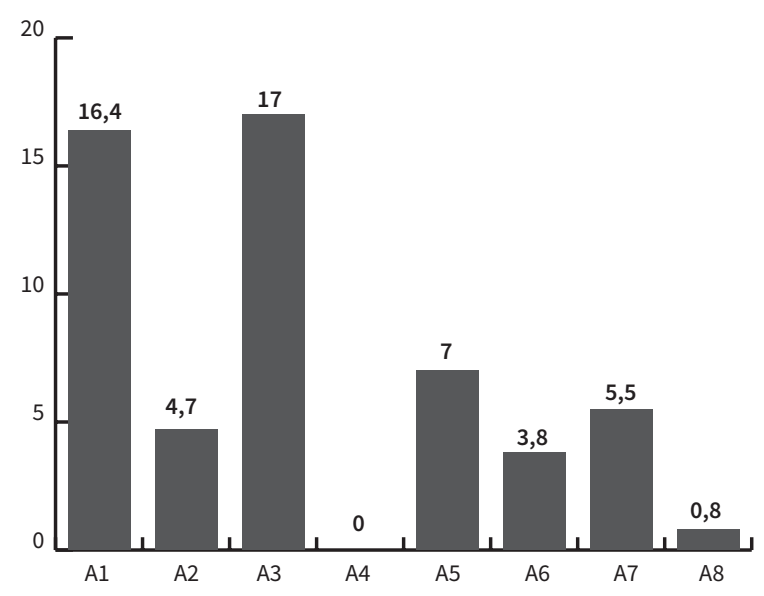

Figura 5. Porcentaje de páginas dedicadas al estudio de las especies no carismáticas invertebradas en cartillas de Escuela Nueva 2008

A1: Ciencias naturales y educación ambiental 2, módulo 1; A2: Ciencias naturales y educación ambiental 2, módulo 2; A3: Ciencias naturales y educación ambiental 3, módulo 1; A4: Ciencias naturales y educación ambiental 3, módulo 2; A5: Ciencias naturales y educación ambiental 4, módulo 1; A6: Ciencias naturales y educación ambiental 4, módulo 2; A7: Ciencias naturales y educación ambiental 5, módulo 1; A8: Ciencias naturales y educación ambiental 5, módulo 2 .

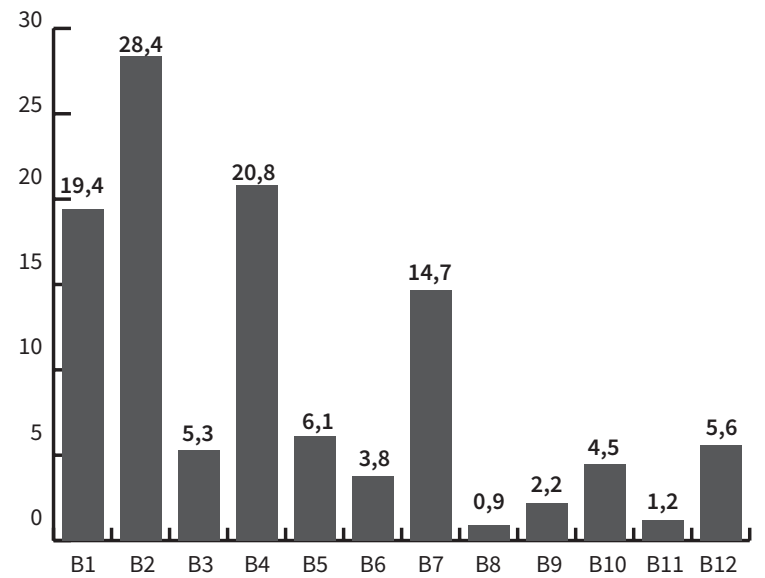

Figura 6. Porcentaje de páginas dedicadas al estudio de las especies no carismáticas invertebradas en cartillas de Escuela Nueva 1996

B1: Ciencias naturales y medio ambiente 2, primera cartilla; B2: Ciencias naturales y medio ambiente 2, segunda cartilla; B3: Ciencias naturales y medio ambiente 2, tercera cartilla; B4: Ciencias naturales y medio ambiente 3, primera cartilla; B5: Ciencias naturales y medio ambiente 3 , segunda cartilla; B6: Ciencias naturales y medio ambiente 3 , tercera cartilla; B7: Ciencias naturales y medio ambiente 4, primera cartilla; B8: Ciencias naturales y medio ambiente 4, segunda cartilla; B9: Ciencias naturales y medio ambiente 4 , tercera cartilla; B10: Ciencias naturales y medio ambiente 5, primera cartilla; B11: Ciencias naturales y medio ambiente 5 , segunda cartilla; B12: Ciencias naturales y medio ambiente 5, tercera cartilla.

\section{Presencia de los conceptos en las guías de aprendizaje de Escuela Nueva}

En relación con la profundidad en la cual se desarrollaron los conceptos, se observó que en la mayoría de las guías de 2008 se trabajan en la sección A (figura 7), donde se incluyen las actividades básicas, en su mayoría se muestran imágenes de animales, tanto vertebrados como invertebrados, que son una especie de introducción al tema central. Seguido de una sección B, que incluía las actividades de práctica, en ellas se encontraban (en algunos casos) lecturas sobre individuos, actividades a desarrollar y textos que explicaban temáticas. Donde menos se involucra el concepto es en la sección C, denominada "Actividades de aplicación", donde se proponen a los estudiantes algunos cuestionamientos para que los resuelvan junto a sus familias.

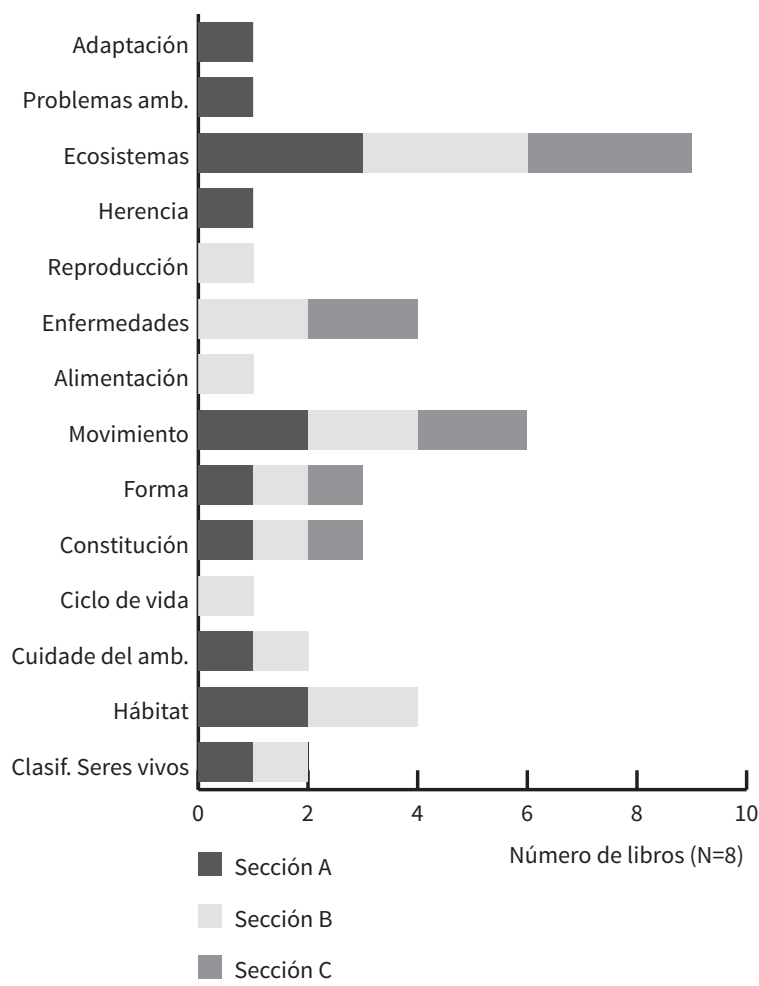

Figura 7. Presencia de los conceptos en las guías de aprendizaje de Escuela Nueva 2008

Mientras que, en las cartillas de 1996, se observó que los conceptos, en su mayoría, están abarcados en la sección A (figura 8), denominada "Construyamos conocimiento". Además, se especifica que estas actividades están diseñadas para el trabajo individual; en su mayoría se muestran imágenes de animales, tanto vertebrados como invertebrados, que son una especie de introducción al tema central. Seguido de una sección B, que incluía las lecturas 
y se indica que es trabajo en grupo, en ellas se abarcan diversidad de temas, en su mayoría historias sobre algunos individuos.

En una menor proporción se encuentra la sección C, denominada "Practiquemos" y es un trabajo individual, estas actividades en su mayoría están diseñadas con base en lecturas programadas que se refieren a las temáticas de la unidad. Por último, la sección $\mathrm{D}$, denominada "Actividades libres", incluye el trabajo sobre especies invertebradas (se encuentra solo en 4 libros de texto).

Por otro lado, se destaca que el concepto de biodiversidad es clave para la comprensión de los procesos biológicos que se llevan a cabo dentro de los ecosistemas (Campos et al., 2012; Mazas y Fernández, 2016), y se encuentra de manera superficial e inmerso en el tema de ecosistemas.

Según Caurín (1999), el estudio de los conceptos por sí solos produce cambios poco significativos en las actitudes, al igual que la realización solamente de actividades destinadas a fomentar el cambio de las mismas. El cambio se produce cuando se combinan adecuadamente los conceptos y las actividades, es decir, cuando a las nociones teóricas de los conceptos se les añaden las aplicaciones prácticas de las actividades.

Al respecto se encuentra su vinculación, pero hace falta el enfoque a la conservación de especies invertebradas y en general a biodiversidad. De aquí nace, entonces, la necesidad de producir, para el docente, ayudas curriculares que estén más enfocadas en este tipo de contenidos.

\section{Consideraciones finales}

Como resultado de esta investigación, se evidenció que los docentes ven los libros de texto proporcionados por el MEN Como recursos importantes para la enseñanza de las ciencias naturales, puesto que contribuyen al aprendizaje en el aula. Sin embargo, consideran importante abordar estos textos con el apoyo de otros libros que completen y refuercen los conceptos abordados.

Las entrevistas realizadas a los docentes permitieron constatar que no hay claridad en cuanto al concepto de especies no carismáticas. No obstante, al realizar el ejercicio de cambiar el sentido de la pregunta, se encontró que conocen el concepto de forma implícita dentro de temas aún más globales que son enseñados en el aula, por ejemplo, reproducción, ecosistema, clasificación de los seres vivos, entre otros.

El tratamiento de contenidos referidos a especies no carismáticas invertebradas en los libros de texto no se abarca de manera individual, sino que está implícito en otras temáticas, como la reproducción, relaciones de los seres vivos, entre otros. Se evidencian muy pocas actividades que fomenten la observación del entorno; por ende, se sugiere plantear actividades en las que el estudiante pueda efectuar un análisis de características de individuos invertebrados en la región, investigaciones sobre especies en la región, visitas a museos, entre otras. De igual forma, los libros de texto muestran imágenes de animales antropomorfizados con fines decorativos.

El tema de especies no carismáticas invertebradas se encuentra en un mayor número de páginas en las guías del 2008 en el módulo 1 de la guía de ciencias naturales 3 , mientras que, en las del año 1998, se encontró un mayor número de páginas en la segunda cartilla de la guía de ciencias naturales y medio ambiente 2 . En relación con la profundidad en la cual se desarrollaron los conceptos, se observó que en la mayoría de las guías de 2008 se trabajan en la sección A, donde incluye las actividades básicas, en su mayoría se muestran imágenes de animales, tanto vertebrados como invertebrados, que son una especie de introducción al tema central.

\section{Referencias}

Arango, X., Rozzi, R., Massardo, F., Anderson, C., e Ibarra, T. (2007). Descubrimiento e implementación del pájaro carpintero gigante (Campephilus magellanicus) como especie carismática: una aproximación biocultural para la conservación en la reserva de biosfera cabo de hornos. Magallania, 35(2), 71-88.

Ballouard, J, Provost, G., Barré, D., y Bonnet, X. (2012). Influence of a field trip on the attitude of schoolchildren toward unpopular organisms: an experience with snakes. Journal of Herpetology, 46(3), 423-428.

Bermúdez, G., De Longhi, A., Díaz, S., y Gavidia, V. (2014). La transposición del concepto de diversidad biológica. Un estudio sobre los libros de texto de la educación secundaria española. Enseñanza de las Ciencias, 32(3), 285-302.

Calderero, J. F. (2003). Estudio de libros de texto de ciencias de la naturaleza mediante análisis cuantitativo basado en la teoría de los grafos (tesis doctoral). Universidad Complutense de Madrid, Madrid. Disponible en http://biblioteca.ucm.es/tesis/edu/ ucm-t26700.pdf

Campos, C., Greco, S., Ciarlante, J., Balangione, M., Bender, J., Nates, J., Lindemann-Matthies, P. 
(2012). Students' familiarity and initial contact with species in the Monte desert (Mendoza, Argentina). Journal of Arid Environments, 82, 98-105. Dol: 10.1016/j.jaridenv.2012.02.013.

Caurín, C. (1999). Análisis, evaluación y modificación de actitudes en Educación Ambiental (tesis doctoral). Universitat de València. Valencia, España.

Dikmenli, M., Çardak, O., y Öztaş, F. (2009). Conceptual Problems in Biology-Related Topics in Primary Science and Technology Textbooks in Turkey. International Journal of Environmental \& Science Education, 4, 429-440.

Franco, A., Castellanos, P., y Medellín, F. (2010). Una mirada al contenido didáctico del concepto adaptación biológica en libros de texto de biología de 6. y 9..$^{\circ}$ grados de educación básica. Bio-grafía: Escritos sobre la Biología y su Enseñanza, 3(4), 1-24.

Gil, M.-J., y de la Gándara, M. (1995). El lenguaje oculto en los libros de texto. Ejemplo "El caso de la adaptación de los seres vivos" (2. ${ }^{\circ}$ ciclo Eso). Aula de Innovación Educativa, 43, 35-39.

Guerra, M., y López, D. (2011). Las actividades incluidas en el libro de texto para la enseñanza de las ciencias Naturales en sexto grado de primaria. Análisis de objetivos, procedimientos y potencial para promover el aprendizaje. Revista Mexicana de Investigación Educativa, 16(49), 441-470.

Henno, I., y Reiska, P. (2010). Difficulty of texts in upper-secondary school biology textbook - using concept maps for analyzing students new knowledge. Making Learning Meaningful Proc. of Fourth Int. Conference on Concept Mapping, Viña del Mar, Chile.

Jiménez, S. (2013). Análisis y evaluación de libros de texto de educación primaria (tesis de grado). Universidad de Navarra. Navarra, España.

Jones, S., Bateman, I., Kontoleon, A., Ferrini, A., Burgess, N., y Turner, K. (2012). Stated preferences for tropical wildlife conservation amongst distant beneficiaries: Charisma, endemism, scope and substitution effects. Ecological economics, 78, 9-18.

López-Valentín, D., y Guerra-Ramos, M. (2013). Análisis de las actividades de aprendizaje incluidas en libros de texto de ciencias naturales para educación primaria utilizados en México. Enseñanza de las Ciencias Revista de Investigación y Experiencias Didácticas, 31(2), 173-191.
Losey, J., y Vaughan, M. (2006). The Economic Value of Ecological Services Provided by Insects. BioScience, 56, 4.

Matthews, R., Flage, L., y Matthews, J. (1997). Insects as teaching tools in primary and secondary education. Annual Review Of Entomology, 42(1), 269.

Gracia, M. V. M. (2003). Análisis del contenido de genética en textos de educación no universitaria. Revista Interuniversitaria de Formación del Profesorado, 17(1), 207-208.

Mateos, A. (1998). Concepciones sobre algunas especies animales: ejemplificaciones del razonamiento por categorías. Dificultades de aprendizaje asociadas. Enseñanza de las Ciencias: Revista de Investigación y Experiencias Didácticas, 16(1), 147-157.

Mazas, B., y Fernández, R. (2016). El concepto de bienestar animal en el currículo de Secundaria Obligatoria y en los libros de texto de ciencias. Revista Eureka sobre Enseñanza y Divulgación de las Ciencias, 13(2), 301-314.

Mckinney, M. (2002). Urbanization, Biodiversity, and Conservation. BioScience, 52, 10.

New, T. R. (2011). Launching and steering flagship Lepidoptera for conservation benefit. Journal of Threatened Taxa, 3(6), 1805-1817.

Occelli, M., Valeiras, N., y Bernardello, G. (2015). La biotecnología en libros de texto de escuela secundaria: un análisis de los libros utilizados en Córdoba (Argentina). Revista Electrónica de Investigación en Educación en Ciencias, 10(1), 33-45.

Prokop, P., Medina, W., Coleman, J., Fedor, P., Fancovicová, J., y Murat, Ö. (2016). Tolerance of Frogs among High School Students: Influences of Disgust and Culture. Eurasia Journal of Mathematics, Science \& Technology Education, 12(6), 1499-1505.

Robinson, T. J., Fischer, L., Wiley, D., y Hilton, J. (2014). The impact of open textbooks on secondary science learning outcomes. Educational Researcher, 43(7), 341-351.

Root-Bernstein, M., Douglas, L., Smith, A., Veríssimo, D. (2013). Anthropomorphized species as tools for conservation: utility beyond prosocial, intelligent and suffering species. Biodiversity and Conservation, 22(8), 1577-1589. Dol: 10.1007/ s10531-013-0494-4

Rosenow, N. (2008). Learning to love the Earth... and each other. YC Young Children, 63(1), 10. 
Samways, M. (2005). Insect diversity conservation. Cambridge: The United Kingdom at the University Press.

Schlegel, J., y Rupf, R. (2010). Attitudes towards potential animal flagship species in nature conservation: A survey among students of different educational institutions. Journal for Nature Conservation, 18, 278-290.

Snaddon, J., Turner, E., y Foster, W. (2008). Children's Perceptions of Rainforest Biodiversity: Which Animals Have the Lion's Share of Environmental
Awareness? PLoS ONE, 3(7), 2579. Dol: 10.1371/journal.pone.0002579

Solaz, J. (2010). La naturaleza de la ciencia y los libros de texto de ciencias: una revisión. Educación xxı, 13(1), 65-80.

Torres, N., y Medina, N. (2014). Representaciones en estudiantes de básica primaria acerca de especies carismáticas y no carismáticas en instituciones educativas rurales y urbanas. Bio-grafía. Escritos sobre la Biología y su Enseñanza, 7, 21-32. 